\title{
TV/Series
}

1 | 2012

Les Séries télévisées américaines contemporaines :

entre la fiction, les faits, et le réel

\section{Les séries historiques entre la fiction et le réel : quand les scénaristes rivalisent avec les historiens}

Ioanis Deroide

\section{OpenEdition}

\section{Journals}

Electronic version

URL: http://journals.openedition.org/tvseries/1038

DOI: $10.4000 /$ tvseries.1038

ISSN: 2266-0909

Publisher

GRIC - Groupe de recherche Identités et Cultures

Electronic reference

Ioanis Deroide, «Les séries historiques entre la fiction et le réel : quand les scénaristes rivalisent avec les historiens », TV/Series [Online], 1 | 2012, Online since 15 May 2012, connection on 10 December 2020. URL : http://journals.openedition.org/tvseries/1038 ; DOI : https://doi.org/10.4000/tvseries. 1038 


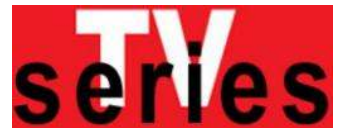
Les séries historiques entre la fiction et le réel :
quand les scénaristes rivalisent avec les historiens
Ioanis DEROIDE

Cet article explore les liens entre les récits que construisent les historiens dans leurs livres et ceux que nous proposent les séries télévisées historiques afin de mettre en lumière comment les seconds peuvent concurrencer les premiers. Sa première partie montre les atouts dont disposent les auteurs de fictions télévisées pour traiter du passé de manière particulièrement vivante et riche, plus encore qu'au cinéma. Sa deuxième partie établit que les séries dites historiques relèvent davantage d'un travail de mémoire que d'histoire puisque justement le passé est saisi comme vivant dans une démarche où l'affect l'emporte sur la raison. Sa troisième partie essaie de définir ce que serait une série véritablement " historienne ", c'est-à-dire construite selon un regard similaire à celui que l'historien porte sur l'objet de son étude.

[In history] what is [...] inferred is essentially something imagined. [...] We find ourselves obliged to imagine Caesar as having travelled from Rome to Gaul when we are told that he was in these different places at these successive times.

[...] It is this activity which, bridging the gaps between what our authorities tell us, gives the historical narrative or description its continuity ${ }^{1}$.

« That's the way they spoke, » [David Milch] said. «I researched the show a good, long time - over a year - and went through a tremendous amount of primary material. And the one thing upon which everyone agrees was the profanity and obscenity was astounding.[...] » Note that Milch said "primary material». He's talking about accounts of Deadwood in letters and diaries from the time in which his show is set and oral histories collected by the Library of Congress' Living Memory project².

$\mathrm{U}$ n historien qui parle d'imagination, un scénariste qui parle d'archives. Cette confusion, au moins apparente, n'est pas nouvelle, et l'on n'a pas attendu l'invention de la télévision pour relever les points communs entre l'histoire des historiens et celle des auteurs de fiction, a fortiori quand il s'agit de fictions historiques.

${ }^{1}$ R. G. Collingwood, The Idea of History. Oxford, Oxford University Press, 1946, p. 241

[« Epilegomena 2: « The Historical Imagination », p. 232-249].

${ }^{2}$ Noel Holson, « Welcome to the vile, vile West », NY Newsday, 21 mars 2004. 
On peut rappeler brièvement quatre similitudes. D'abord, il est admis que l'œuvre de l'historien, comme celle du romancier, du scénariste, est un discours tenu sur le passé, et non la résurrection du passé lui-même. Ensuite, dans les deux cas, ce discours est formulé au présent et porte la marque d'un sujet irrémédiablement séparé de son objet. Troisièmement, il s'intéresse à des êtres et des événements particuliers et non - comme les sciences expérimentales - à des lois universelles. Enfin, il prend la forme d'une narration, d'un récit toujours lacunaire et irrégulier, fait de pauses et d'ellipses.

Cette question des rapports entre histoire et fiction suscite depuis quelques années un regain d'intérêt qui s'est traduit par plusieurs publications ${ }^{3}$.

Les amateurs de séries télévisées américaines sont bien placés pour prendre part à cette discussion, surtout depuis que les fictions historiques ont trouvé sur les écrans des chaînes du câble une place de choix, c'est-à-dire depuis 2001, année de la première diffusion de Band of Brothers sur HBO. Une quinzaine de séries ou mini-séries ont été programmées depuis et cette vogue semble se poursuivre: une deuxième saison de The Borgias (Showtime, 2011-) a été commandée après le succès de la première, Boardwalk Empire (HBO, 2010-) puis Mad Men (AMC, 2007-) feront prochainement leur retour, et de nouvelles productions, comme Hell on Wheels (AMC), ou Magic City (Starz) sont également annoncées.

L'ambition de cet article est de réussir à montrer que les scénaristes des séries télévisées américaines contemporaines sont les rivaux les plus sérieux des historiens. En effet, si les séries historiques offrent une expérience du passé vivante et complète, ce n'est pas seulement parce qu'elles s'inscrivent dans une démarche mémorielle ; c'est aussi parce que la forme de la série américaine contemporaine elle-même présente des points communs avec l'étude historienne.

\section{Une expérience du passé particulièrement riche}

Les séries historiques américaines d'aujourd'hui, du moins les plus marquantes d'entre elles, semblent bien proposer une vision du passé plus riche que celle des historiens. Deux arguments peuvent être avancés pour appuyer cette proposition.

Par l'usage de l'image et du son, bientôt par la sensation de relief, les séries ont le pouvoir de reconstituer le passé, c'est-à-dire non pas de le ressusciter - chose impossible - mais de le rendre

3 Annales. Histoires, Sciences sociales, $65^{\mathrm{e}}$ année, $\mathrm{n}^{\circ} 2$ ( « Savoirs de la littérature »), marsavril 2010. Mona Ozouf, " Historien et romancier : actualité d'un vieux débat », conférence donnée à la Bibliothèque Nationale de France le 8 mars 2011. Le Débat, $\mathrm{n}^{\circ} 165$ ( «L'Histoire saisie par la fiction »), mai-juin 2011. Critique, $\mathrm{n}^{\circ} 769-770$ ( « Traces de Carlo Ginzburg »), juin-juillet 2011. 
éminemment sensible à nos perceptions contemporaines, ce qui peut apparaître comme le rêve de tout historien : que le monde passé, à jamais disparu, reprenne forme devant nos yeux.

Or, comme le rappelle Antoine de Baecque, « dès le cinéma de Griffith, filmer l'histoire consistait pour le cinéma à rivaliser d'ambition épique avec celle-ci. Comme si reconstituer l'histoire c'était, d'une certaine manière, lui faire concurrence par le cinéma 4 ». Une telle démarche, qui favorise les sujets épiques servis par des superproductions, se retrouve aussi à la télévision: on pense évidemment à Band of Brothers (HBO, 2001) et The Pacific (HBO, 2010), deux projets directement inspirés de l'expérience cinématographique de Saving Private Ryan (1998). Quand leurs moyens financiers sont plus modestes, les séries se souviennent qu'elles ont d'autres parents que le cinéma : le théâtre et la radio. Elles n'abandonnent pas pour autant leur rêve de reconstitution mais elles le cantonnent à de plus petits espaces : ainsi, le monde de Mad Men est-il presque exclusivement constitué d'intérieurs domestiques, de bureaux, de mobilier et de costumes. Ces choix de décors sont bien sûr indissociables des choix de périodes et de regard historique : histoire des mentalités pour Mad Men, histoire sociale pour Deadwood (HBO, 2004-2006), histoire militaire pour The Pacific.

Dans tous les cas, la série possède un avantage sur d'autres formes d'expression : le « retour de l'identique 5 ». La fréquentation des mêmes lieux, qu'il s'agisse du forum romain, du Gem Saloon de Deadwood ou du boardwalk d'Atlantic City nous les rend de plus en plus familiers, épisode après épisode. Elle favorise une immersion sur le long terme dans l'époque passée là où le cinéma, qui peut certes nous plonger plus rapidement dans un autre monde grâce à ses salles obscures et ses grands écrans, doit aussi nous en laisser ressortir plus vite, avant même peut-être que le dépaysement ait produit tous ses effets. Pas de regret de ce type dans la plupart des séries historiques : au contraire, on prend le risque d'une usure d'un décor trop souvent vu, ce qui conduira à en changer à la faveur d'un rebondissement scénaristique comme la recréation de l'agence de publicité à la fin de la saison 3 de Mad Men.

Le second argument est double: il s'agit du recours systématique aux intrigues dramatiques et aux personnages archétypaux qui en sont les acteurs. Ce recours produit des significations plus accessibles.

Les notions d'intrigue et de personnage ne sont pourtant pas étrangères à l'histoire, bien au contraire.

${ }_{4}^{4}$ Antoine de Baecque, Histoire et cinéma, Paris, Cahiers du Cinéma / SCÉRÉN-CNDP, Coll. «Les petits Cahiers », 2008, p. 11-12.

5 Umberto Eco, « Innovation et répétition : entre esthétique moderne et post-moderne »,

Réseaux, vol. 12, nº8, 1994, p. 15 [p. 9-26]. 
[L'historien doit d'abord] configurer son sujet. Il ne le trouve jamais tout fait, il le construit. Il le façonne par un acte inaugural et constitutif qu'on peut désigner comme une mise en intrigue (emplotment dans la littérature américaine). Celle-ci porte notamment sur les personnages et les scènes. Elle est choix des acteurs et des épisodes ${ }^{6}$.

Quelle différence demeure, à ce stade, entre l'historien et le scénariste ? La question se pose d'autant plus sérieusement que les historiens contemporains s'adonnent avec passion à un genre où le personnage est central : la biographie.

On peut parler aujourd'hui d'une fièvre biographique qui répond aux attentes d'un public de plus en plus friand d'une dimension proprement existentielle dans les représentations qu'on peut se faire du passé7.

Cette «dimension existentielle », n'est-ce pas précisément ce que The Tudors (Showtime, 2007-2010), Mad Men ou Boardwalk Empire prétendent mettre en avant ? Ces trois séries peuvent en effet être vues comme des biographies télévisuelles. Elles résument d'ailleurs la diversité du genre eu égard à la notion de personnage historique : biographie d'un "grand homme » - le roi Henri VIII dans The Tudors, biographie d'un personnage fictif (Nucky Thompson) très fortement inspiré d'un personnage réel (Nucky Johnson) dans Boardwalk Empire, biographie enfin d'un personnage tout à fait fictif, qui plus est dissimulateur et affabulateur, dans le cas de Don Draper.

Il y a pourtant des différences, en matière d'intrigue, entre l'histoire et la série, qui sont à l'avantage de la seconde. Premièrement, dans la série télévisée, où le récit occupe toute la place, la densité d'action est bien plus élevée que dans les livres d'histoire. Ceux-ci peuvent en effet prendre la forme d'un tableau, par exemple dans $L a$ Société féodale, de Marc Bloch, ou d'un commentaire comme l'ouvrage de François Furet, Penser la Révolution française ${ }^{8}$. Deuxièmement, le recours aux personnages permet de faire saisir simplement une période, son organisation sociale, sa diversité. Plusieurs personnages de Mad Men remplissent clairement cette fonction : par exemple, les trois principaux personnages féminins, Betty, Joan et Peggy représentent autant de types de la condition féminine des classes moyennes urbaines au début des années 1960. De même, dans Rome (HBO, 2005-2007), Pullo le prolétaire et Vorenus le petit patron sont

\footnotetext{
${ }^{6}$ Antoine Prost, Douze leçons sur l'histoire, Paris, Éditions du Seuil, Coll. « Points Histoire », 1996, p. 245 .

7 François Dosse, L'Histoire, Paris, Armand Colin, Coll. "Cursus », $2^{\mathrm{e}}$ édition, 2010, p. 96

${ }^{8}$ Nous reprenons la typologie et les exemples d'Antoine Prost. Prost, p. 239-242.
} 
des personnages fictifs bien utiles pour nous faire comprendre les tensions politiques et sociales qui parcourent la société romaine à la fin de la République.

L'argument de l'intrigue et du personnage souligne à nouveau une certaine supériorité de la série sur le cinéma, qui tient à la durée. Une durée plus longue, cela signifie d'abord davantage d'exhaustivité : les trente-huit épisodes des Tudors nous offrent un récit plus complet et détaillé du règne d'Henri VIII que chacun des longs-métrages (une quinzaine environ) consacrés à ce célèbre monarque. Ce temps plus long ne rend pas la série nécessairement meilleure que ces films mais il permet d'intégrer au récit un plus grand nombre d'événements historiques, de mieux expliquer et contextualiser la politique menée par le roi. Or, The Tudors, si elle n'hésite pas à racoler le spectateur par de nombreuses scènes de violence et de sexe, ne recule pas non plus devant la difficulté d'évoquer la Réforme protestante ou la diplomatie européenne.

De plus, du fait de la durée des séries, les personnages peuvent incarner, non seulement un certain état de la société ou de l'idéologie, mais aussi leurs évolutions, et celles-ci peuvent être rendues plus finement et de manière plus vraisemblable qu'en une, deux ou trois heures au cinéma. Sur ce point, les séries historiques se démarquent de beaucoup d'autres séries, auxquelles on reproche leurs personnages immobiles, prisonniers d'une formule à succès et condamnés à n'évoluer qu'au ralenti ou en boucle, chaque changement étant immanquablement suivi d'un retour à la normale.

Nous avons donc mis en évidence la richesse, la vie, l'émotion dans le rapport au passé qui caractérisent les séries historiques. A vrai dire, ce lexique fait songer à une approche mémorielle plutôt qu'historique. Et en effet, à bien y regarder, les scénaristes de séries historiques font surtout œuvre de mémoire.

\section{Les séries historiques, des séries mémorielles}

Travail de mémoire, travail d'histoire : les deux démarches ne doivent pas être confondues car elles diffèrent grandement dans leurs principes.

La mémoire est la vie, toujours portée par des groupes vivants et à ce titre, elle est en évolution permanente, ouverte à la dialectique du souvenir et de l'amnésie, inconsciente de ses déformations successives, vulnérable à toutes les utilisations et manipulations, susceptible de longues latences et de soudaines revitalisations. L'histoire est la reconstruction problématique de ce qui n'est plus. Parce qu'elle est affective et magique, la mémoire [...] se nourrit de souvenirs flous, téléscopants, globaux ou flottants, particuliers ou symboliques, sensible à tous les transferts, écrans, censure ou projections. L'histoire, parce que opération intellectuelle et laïcisante, appelle analyse et discours 
critique9.

Les historiens doivent donc être animés d'un même souci de rigueur, de rationalité, de neutralité, qui peut heurter la mémoire : "L'histoire est délégitimation du passé vécu ${ }^{10}$ ».

Dans le cas des séries historiques, on se trouve bien souvent, semble-t-il, du côté de ce passé vécu, et même à double titre puisqu'à la mémoire de la période considérée vient s'ajouter celle de ses représentations.

L'exemple le plus frappant est celui de la Deuxième Guerre mondiale qui a fait l'objet depuis une petite quinzaine d'années de productions relevant explicitement d'une démarche mémorielle. Dans la catégorie documentaire, les quatorze heures de The War (PBS, 2007), constituent sans conteste la production la plus ambitieuse. Dans celle des fictions, Band of Brothers puis The Pacific ont inauguré un nouveau traitement du conflit, sous la forme de "feuilletons de prestige $^{11}$ » où l'expérience confuse du combat que font les soldats est contrebalancée par des contextualisations didactiques, qui donnent toute leur place aux témoins de cette période. Dans les deux cas, le souci d'authenticité, martelé par le discours promotionnel, conduit, nous dit-on, à réviser les représentations communément admises et à admettre que le Débarquement de Normandie, voire la bataille de Tenaru, peuvent soutenir la comparaison avec les pires heures de la guerre du Vietnam, considérée jusqu'alors comme un summum d'horreur et d'absurdité.

Ce discours s'explique en grande partie par l'époque : le G.I. qui avait 20 ans sur les plages de Normandie en 1944 en a 77 au moment de la première diffusion de Band of Brothers. De manière plus générale, la disparition progressive des anciens combattants de la Deuxième Guerre mondiale rend plus nécessaire, semble-t-il, la remémoration de leurs actions : on entre, dès le milieu des années 1990, dans " l'ère du témoin ${ }^{12}$ »: " Au témoignage spontané a succédé l'impératif social de mémoire. Le survivant se doit d'honorer un " devoir de mémoire " auquel il ne peut moralement se dérober » ${ }^{13}$. Ces propos concernent les rescapés de la Shoah, mais on peut les appliquer ici aux combattants américains.

Dans nos deux feuilletons, les témoins sont omniprésents. On les trouve à la source puisque les mémoires de soldats constituent le

9 Pierre Nora, «Entre mémoire et histoire», in Les Lieux de mémoire, tome 1, « La République », éd. Pierre Nora, Paris, Gallimard, coll. «Bibliothèque illustrée des histoires», 1984, p. XIX.

${ }_{10}$ Op. cit., p. XX.

${ }^{11}$ Nous empruntons cette expression à Stéphane Benassi, « Sérialité(s) », in Décoder les

séries télévisées, éd. Sarah Sépulchre, Bruxelles, De Boeck, 2011, p. 85.

${ }^{12}$ Annette Wieviorka, L'Ère du témoin, Paris, Plon, 1998.

13 Op. cit., p. 160. 
matériau principal sur lequel ont travaillé les scénaristes ${ }^{14}$. On les suit tout au long du récit à travers les personnages qui reprennent leur nom dans la fiction. On les regarde et on les écoute dans de petits segments placés au début de chaque épisode : ils s'y expriment face à la caméra pour partager leurs souvenirs, comme d'autres le font via les écrans disposés dans les nombreux musées et mémoriaux consacrés à la guerre aux États-Unis et en Europe. Et s'ils sont encore vivants au moment de la diffusion, ils sont honorés en héros familiers: les survivants de la Easy Company de Band of Brothers sont ainsi présents en duplex lors de la cérémonie des Emmy Awards en septembre 2002 et l'assistance leur décerne une standing ovation.

Une telle attention portée aux témoins, au caractère sacré de leur parole, sert efficacement un projet mémoriel car l'émotion de l'octogénaire se remémorant les heures glorieuses et tragiques de sa jeunesse ne peut laisser indifférent, mais elle méconnaît les règles de prudence qui doivent présider au recueil du souvenir quand on souhaite l'utiliser pour mieux connaître l'histoire.

Le témoin des années quatre-vingt-dix, celles de l'explosion du témoignage, est un homme ou une femme d'un certain âge. La tonalité de son témoignage est largement influencée par le bilan qu'il peut faire de son existence. [...] Or, la personne interviewée l'est en raison de son expérience dans les années de la Seconde Guerre mondiale, même s’il lui est demandé d'évoquer l'avant et l'après. Ce n'est donc pas un « récit de vie » qui est recueilli, mais, et la demande est claire, le récit de sa vie informé par cette période dont il est ainsi postulé qu'elle est rupture fondamentale. Toute l'histoire de l'individu se trouve ainsi nouée autour de ces années de sa vie en vertu d'un pur postulat : que cette expérience a été l'expérience décisive d'une vie.

Et en effet, les auteurs de The Pacific sont convaincus que la bataille de Peleliu (septembre-novembre 1944) fut une "expérience décisive » pour leurs personnages Eugene Sledge et Robert Leckie. C'est pourquoi, comme dans Band of Brothers, ils prennent le temps d'évoquer l'avant et l'après-guerre de leur héros : ils veulent nous faire mesurer combien ces hommes ont été changés à tout jamais. Dans le même ordre d'idées, ils nous présentent des personnages secondaires, moins forts ou moins chanceux, brisés psychiquement par l'horreur des combats, voire poussés au suicide (The Pacific, 1.4).

Cette insistance à souligner les terribles épreuves traversées par les soldats participe d'un grand élan médiatique d'admiration qui s'exprime depuis plus d'une dizaine d'années, aux États-Unis, à l'égard des aînés ayant combattu de 1941 à 1945, honorés comme la «greatest

\footnotetext{
${ }^{14}$ Par exemple : Eugene Sledge, With The Old Breed: at Peleliu and Okinawa, Novato,
} Presidio Press, 1981, qui fut beaucoup utilisé pour The Pacific mais aussi pour The War. 
generation ${ }^{15} »$.

Mémoire de la guerre, donc, mais aussi mémoire des films de guerre, jugés trop propres et statiques. De la même manière, Bruno Heller, le co-créateur et producteur exécutif de Rome, tenait à expliquer que la ville qu'ils avaient reconstituée n'était pas celle des péplums.

"You rarely see onscreen the complexity and color that was ancient Rome, » says co-creator, executive producer and writer Bruno Heller. "It has more in common with places like Mexico City and Calcutta than quiet white marble. Rome was brightly colored, a place of vibrant cruelty, full of energy, dynamism and chaotic filth ${ }^{16}$. »

David Milch revendique une prise de distance similaire dans le cas de Deadwood.

As David Milch [...] is quick to point out, the series is not a Western in the traditional sense of the genre. In fact, Milch said he devoted no time to watching old Westerns as a part of his research.

" The genre itself had no particular attraction for me, » Milch said. "I like to think of this story as set in the West, rather than a Western ${ }^{17}$.

Toutefois, la mémoire ne conduit pas toujours au rejet des représentations antérieures, d'ailleurs souvent exagéré pour faire ressortir le caractère novateur de la dernière production en date. Ainsi, dans Carnivàle (HBO, 2003-2005), série qui se déroule pendant la Grande dépression des années 1930, un hommage est rendu dans le premier épisode à Grapes of Wrath de John Ford (1940) : la scène où le jeune Ben Hawkins est expulsé de sa ferme est en effet une citation de celle qui voit Muley Graves connaître le même sort au début du film.

Au total, un survol des grandes séries historiques des chaînes du câble nous fait prendre conscience que la plupart d'entre elles souhaitent avant tout réactualiser le souvenir des grandes heures du roman national, de la conquête de l'Ouest aux évolutions culturelles des Sixties.

Si l'on élargit l'étude aux séries historiques diffusées sur les

${ }^{15}$ Expression reprise dans le best-seller d'un célèbre journaliste de télévision. Tom Brokaw, The Greatest Generation, New York, Random House, 1998.

${ }^{16}$ " Ciaran Hinds, Kevin McKidd and Lindsay Duncan head the cast of HBO/BBC epic series Rome », dossier de presse, 28 juin 2005.

<www.bbc.co.uk/pressoffice/pressreleases/stories/2005/o8_august/26/rome.shtml>, consulté le 26 juin 2011.

${ }_{17}$ Jessica Feinstein, « Crossing genres, ' Deadwood' dominates », Yale Daily News, 26 mars 2004. <www.yaledailynews.com/news/2004/mar/26/crossing-genres-deadwooddominates/>, consulté le 26 juin 2011. 
networks ces vingt dernières années, la dimension mémorielle apparaît encore plus fortement, les périodes choisies étant plus récentes - les décennies 1960 et 1970 surtout - pour des raisons de coût et de public visé. Dans The Wonder Years (ABC, 1988-1993), la démarche est tout à fait explicite puisque chaque épisode est introduit par la voix over du narrateur adulte qui se souvient de son enfance et de son adolescence. Dans Quantum Leap (NBC, 1989-1993) et Life on Mars (ABC, 20082009), on matérialise la remémoration en faisant effectuer au héros un retour littéral dans le passé sous la forme d'un «voyage dans le temps ». Dans Freaks and Geeks (NBC, 1999-2000), American Dreams (NBC, 2002-2005) ou Swingtown (CBS, 2008), le propos est moins transparent mais tout est fait pour rappeler aux téléspectateurs les difficultés et les bonheurs de leur jeunesse, aussi présentée comme celle de leur pays. Selon le genre du programme concerné, l'accent est mis davantage sur les éléments les plus pittoresques et comiques -dans la sitcom That '7os Show (Fox, 1998-2006) - ou sur les aspects les plus douloureux, comme dans le policier Cold Case (CBS, 2003-2010). Chaque épisode de ce procedural commence par la réouverture de l'enquête sur une vieille affaire de meurtre et c'est un travail de deuil qui nous est proposé.

Ce travail s'articule autour de deux revisites critiques : d'une part, celle des événements marquants ou cachés de l'histoire des ÉtatsUnis [...] et, d'autre part, celle de modes de fonctionnements intolérants. [...] Le téléspectateur a l'impression de vivre ou de revivre, de nommer puis d'enterrer des moments forcément difficiles du passé des ÉtatsUnis $^{18}$.

Pour la saison 2011-2012, deux séries dont le récit débute en 1963 sont annoncées : Pan Am (ABC) et The Playboy Club (NBC). Toutes deux sont présentées par la critique américaine comme de pâles copies de Mad Men proposant une vision hédoniste des années soixante afin d'offrir aux téléspectateurs une compensation symbolique aux frustrations que leur inflige la société américaine de $2011^{19}$. Par où l'on confirme que « la mémoire ne s'accommode que des détails qui la confortent ${ }^{20} »$.

On peut conclure des paragraphes qui précèdent que les séries américaines contemporaines qui appréhendent le passé le font sous l'angle de la mémoire. Pour autant, il ne nous semble pas que l'on doive les ranger exclusivement de ce côté. Nous allons essayer au contraire de montrer qu'au-delà du genre, « historique » ou autre, la forme de la

${ }^{18}$ Laurent Malbrunot, Cold Case. Les Cicatrices du passé, Paris, Alphée, 2010, p. 44-45 ${ }_{19}$ Melissa Maerz, "Longing for a time when bad was good », Los Angeles Times, 22 mai 2011.

${ }^{20}$ Nora, p. XIX. 
série elle-même peut être une forme historienne.

\section{Des séries historiennes}

Pour établir si le raisonnement des scénaristes présente des similitudes avec celui des historiens, partons d'une évidence. Les séries américaines contemporaines se présentent sur plusieurs temporalités : celle de l'épisode, celle de la saison, celle de la série tout entière. Cette pluralité revêt une grande importance aux yeux des sériephiles qui la prennent toujours en compte au moment de formuler leurs avis : " D'accord, il y avait pas mal de mauvais épisodes, mais globalement j'ai trouvé que c'était une bonne saison ${ }^{21}$ ». C'est cette double, voire triple, lecture, érigée en ambition pédagogique, qui a poussé la critique française spécialisée, éclose dans la première moitié des années 1990, à fournir des « modes d'emploi » à ses lecteurs afin qu'ils apprennent à mieux regarder les séries. Ces petites notices, que l'on retrouve dans plusieurs ouvrages ${ }^{22}$, prennent bien soin de distinguer les saisons, de signaler les temps forts et les passages à vide, de souligner le cas échéant la variété des genres abordés par un même programme d'un épisode à l'autre. Cette éducation a depuis été relayée par de nombreux médias de sorte que le fan de séries de 2011, désormais familier de leur architecture, est devenu à l'instar de son homologue américain un amateur de jeu d'échelles temporelles.

Voilà un point commun avec l'historien, habitué, surtout depuis Fernand Braudel, à distinguer au moins trois durées.

L'histoire traditionnelle attentive au temps bref, à l'individu, à l'événement, nous a depuis longtemps habitués à son récit précipité, dramatique, de souffle court.

La nouvelle histoire économique et sociale met au premier plan de sa recherche l'oscillation cyclique et elle mise sur sa durée [...]. Il y a ainsi, aujourd'hui, à côté du récit (ou du « récitatif » traditionnel), un récitatif de la conjoncture qui met en cause le passé par larges tranches : dizaines, vingtaines ou cinquantaines d'années.

Bien au delà de ce second récitatif se situe une histoire de souffle plus soutenu encore, d'ampleur séculaire cette fois : l'histoire de longue, même de très longue durée 23 .

Si l'historien distingue ces trois temps, c'est pour donner toute leur place à des évolutions autres que politiques ou diplomatiques, pour ouvrir le champ de la recherche aux dynamiques économiques,

${ }^{21}$ Yerno, « Les Adieux », in La Ligue des Téléspectateurs extraordinaires [en ligne]. $<$ www.asuivre.org/ archives/lte/article.php3?id article=4436>, consulté le 29 juin 2011. ${ }^{22}$ Le premier est Alain Carrazé, Christophe Petit, Les grandes séries américaines des origines à 1970, Paris, Huitième Art, 1994.

${ }^{23}$ Fernand Braudel, « Histoire et Sciences sociales : La longue durée », in Annales. Économies, sociétés, civilisations. $13^{\mathrm{e}}$ année, $\mathrm{n}^{\circ} 4$, 1958. p 727 [p. 725-753]. 
sociales, culturelles, pour enfin présenter une histoire des civilisations qui nécessite d'embrasser de larges portions de ces quelques millénaires qui constituent l'histoire des hommes. L'idée sous-jacente à cette prise de hauteur est que les réalités les plus profondes se dévoilent seulement sur la longue durée, ou qu'en tout cas la vérité du jour n'est pas celle de la décennie qui n'est pas celle du siècle.

Les séries présentent aussi un grand écart entre la plus petite unité de compte - l'épisode, ou même, en découpant davantage, l'acte voire la séquence - et la plus grande - la série dans son ensemble : en proportion, cinq saisons sont à un épisode ce qu'un siècle est à un an. Nous retrouvons également dans ces fictions une variété de significations en fonction de l'échelle retenue. Dans The $X$-Files (Fox, 1993-2002), un épisode nous lance à la poursuite du fameux Jersey Devil (1.04), une saison nous fait prendre la mesure - partielle - d'un nébuleux complot qui menace l'humanité mais la série tout entière fait avant tout le récit de la rencontre et de l'union de deux âmes sœurs, Mulder et Scully, personnalités complémentaires et inséparables.

Si la multiplicité des échelles est une condition pour qu'une série soit considérée comme historienne, les séries historiques ne semblent pas pouvoir prétendre à ce titre car elles sont contraintes par leur genre même à une certaine unité de ton réaliste. Si elles choisissent de représenter un passé fantaisiste, elles changent de catégorie, comme c'est le cas pour Spartacus: Blood and Sand (Starz, 2010-) ou sa prequel, Spartacus: Gods of The Arena (Starz, 2011), et plus encore pour Camelot (Starz, 2011-) qui se déroulent respectivement dans une Antiquité et un Moyen-Âge largement réinventés voire mâtinés de merveilleux. Elles ne peuvent pas même s'autoriser, le temps d'un épisode, une excursion dans un territoire fictionnel où la vraisemblance serait trop malmenée, où les repères habituels s'effaceraient. Cette licence, qui a permis par exemple à The $X$-Files d'enrichir son registre, leur est interdite.

D'un autre côté, cette unité de ton des séries historiques, associée à leur brièveté - sur le câble, seules The Tudors et Mad Men ont atteint la quarantaine d'épisodes - plaide en faveur de leur caractère historien car elle tend à en faire des fictions bien closes. Or « toute entreprise historienne se définit par une clôture 24 », c'est-à-dire par l'identification d'un début et d'une fin. Si la question du point de départ du récit est parfois soulevée dans les discours critiques sur les séries, celle du point d'arrivée l'est presque toujours puisque celui-ci est incertain, chargé des attentes croissantes du public au fur et à mesure que se prolonge la durée de vie de la fiction, et souvent décevant en regard de ces attentes. Parfois, il n'existe tout simplement pas, dans le cas des programmes qui sont retirés de l'antenne si brutalement que leur narration reste pour toujours privée de fin.

${ }^{24}$ Prost, p. 243. 
Le récit des séries historiques, quant à lui, est contraint par la chronologie des événements réels, préalablement découpée en périodes nommées par les contemporains et les historiens: la République romaine, la Deuxième Guerre mondiale, etc. Ces séries n'ont donc pas à créer de toutes pièces leur terme, ce qui peut être considéré comme un avantage. Nous savions avant d'avoir vu le dernier épisode que Band of Brothers et The Pacific ne pourraient se prolonger bien au-delà de 1945 et The Tudors devait s'achever à la mort d'Henri VIII. De la même manière, même si le dernier épisode de Boardwalk Empire ou des Borgias n'est pas encore écrit, on peine à imaginer que ces séries puissent se poursuivre longtemps après, respectivement, la fin de la prohibition (1933) et la mort de César Borgia (1507). Certes, savoir quand ces séries finiront ne permet pas de savoir comment elles finiront mais l'étendue des possibles scénaristiques s'en trouve tout de même réduite.

De même, savoir à quel moment du passé le récit trouvera son terme ne permet même pas de prédire à quel moment de l'avenir la série finira d'être produite : on peut choisir de repousser indéfiniment la conclusion d'une série historique en étirant démesurément la période choisie pour y faire tenir un grand nombre d'épisodes. C'est le choix fait, dans les années 1960, par Combat! (ABC, 1962-1967), une série qui suit, cinq saisons durant, des soldats américains libérant le territoire français sans jamais leur faire passer la frontière allemande. Mais, on l'a vu, l'exigence de reconstitution des séries historiques actuelles - et le coût des moyens matériels et humains qui tentent de la satisfaire - s'accommodent mal de telles libertés prises avec le déroulement de la chronologie.

La série historienne est donc celle qui allie un propos tenu à plusieurs échelles et une clôture rigoureuse : la série parfaite, en quelque sorte, d'autant plus que ces deux qualités sont difficilement conciliables.

Tant que les scénaristes et tous ceux qui traduisent en images leurs idées reconstituent le passé, ils créent un spectacle envoûtant mais qui demeure à côté de l'histoire car il ne peut ni ne veut égaler sa rigueur et son exigence de vérité. C'est quand ils poursuivent le double objectif de complexité et de cohérence présenté ci-avant que les auteurs de séries concurrencent le plus sérieusement les historiens parce qu'alors ils racontent des vies comme on raconte l'histoire : avec un début, une fin et différentes focales. Nous aider à penser nos existences comme des histoires, tel n'est pas le moindre mérite des séries télévisées américaines contemporaines. 